\title{
Automated Qualitative Element Analysis of EDS Spectra with Considering Pile- Up Distortions in High Count-Rate Modes
}

\author{
F. Eggert, T. Elam, R. Anderhalt and J. Nicolosi \\ EDAX Inc., a Business Unit of Ametek Materials Analysis, \\ 91 McKee Drive, Mahwah, NJ, 07430
}

The ability to do qualitative analysis (identification of all elements in specimen spectrum) is a major advantage of Energy Dispersive Spectrometers (EDS). The benefit of an EDS detector is the collection of a total spectral distribution in one data acquisition step. Therefore it is well suited to the qualitative analysis question. Many developments of EDS spectrometry models for spectra evaluation have lead to improvements in quantitative analysis, but without similar advances for automated qualitative evaluation. This is questionable, because all quantitative evaluation models rely on an assumption about which elements are present in the specimen. As in past years the analyst has to use line-markers to compare to the acquired spectrum. Occasionally they are supported by spectrum reconstruction tools to aid in recognizing incorrect element identifications visually by comparison to an artificial spectrum. But this is like a trial and error method, not automated and needs some skills and experience with EDS.

A completely new approach for automated element identification in EDS spectra was published recently [1] and is named EXpertID. Basically there are used multiple spectra deconvolution and reconstruction assessments. This is in combination with residual peak searches, integrated standardless quantitative evaluation, computation of analytical errors with a final uncertainty estimation and even parts of spectra simulation steps [2], just the way an experienced analyst would go. This automated evaluation scheme solves the qualitative analytical question for totally unknown specimens, first time with a full standardless evaluation.

The use of Silicon Drift Detectors (SDD) in Electron Microscopes comes with the ability to acquire spectra with very high count rates. But the high count rate spectra are the major challenge with this new detector technology. The spectra contain many pile-up artefacts and are often called 'dirty spectra' from the point of view of normal spectra evaluation. The qualitative evaluation procedure is faced with these artefacts and produce quite often bad or wrong results. Sum peaks are wrongly detected as characteristic element lines and cause 'false-positives' in element identification. The Bremsstrahlung background is affected by smeared pile-up components which can disturb the assessment loops for qualitative analysis, based on reconstruction of the entire spectrum. Therefore a correction is required to retain reliable results. But there are limits in application of the known approaches [3, 4], quickly reached with about $40 \mathrm{kcps}$ count-rates and not possible to overcome without additional knowledge. Overcorrections and oscillations occur and are visible in the corrected spectrum, if used beyond the applicability of the corrections. But this makes issues for automatic element identification, same as the pile-up artefacts before correction.

EXpertID is based on multiple spectra reconstructions. That means, with each reconstruction step also the background is calculated. This estimation for the spectrum background is beneficially usable to support the pile-up correction. In simplified form, all visual correction mistakes are possible to avoid by including the 'known' background with statistically based assessments of pile-up correction results. This makes it even possible to get the pile-up correction self-adjustable, calculated again with each background calculation. Therefore the new idea is to avoid an explicit correction 
after spectra acquisition, but to consider the pile-up effect internally in all qualitative and quantitative iteration loops, also with EXpertID assessments (iteratively). The 'Smart PileUpConsideration' will be introduced for the first time with [5]. The method is an integrated part of spectra evaluation and makes it possible to evaluate 'dirty spectra' with count rates of $100 \mathrm{kcps}$ and even much more, without significantly changes in qualitative (and quantitative) analysis results.

\section{References}

[1] F. Eggert, IOP Conf. Ser.: Mater. Sci. Eng. 7 (2010), 012007

[2] F. Eggert, Microchim Acta 155 (2006), 129

[3] P. J. Statham, Microchimica Acta 155 (2006), 289

[4] T. Elam, R. Anderhalt, J. Nicolosi, Microsc Microanal 14 Suppl.2 (2008), 1260

[5] F. Eggert, T. Elam, R. Anderhalt, J. Nicolosi, to be bublished at EMAS 2011
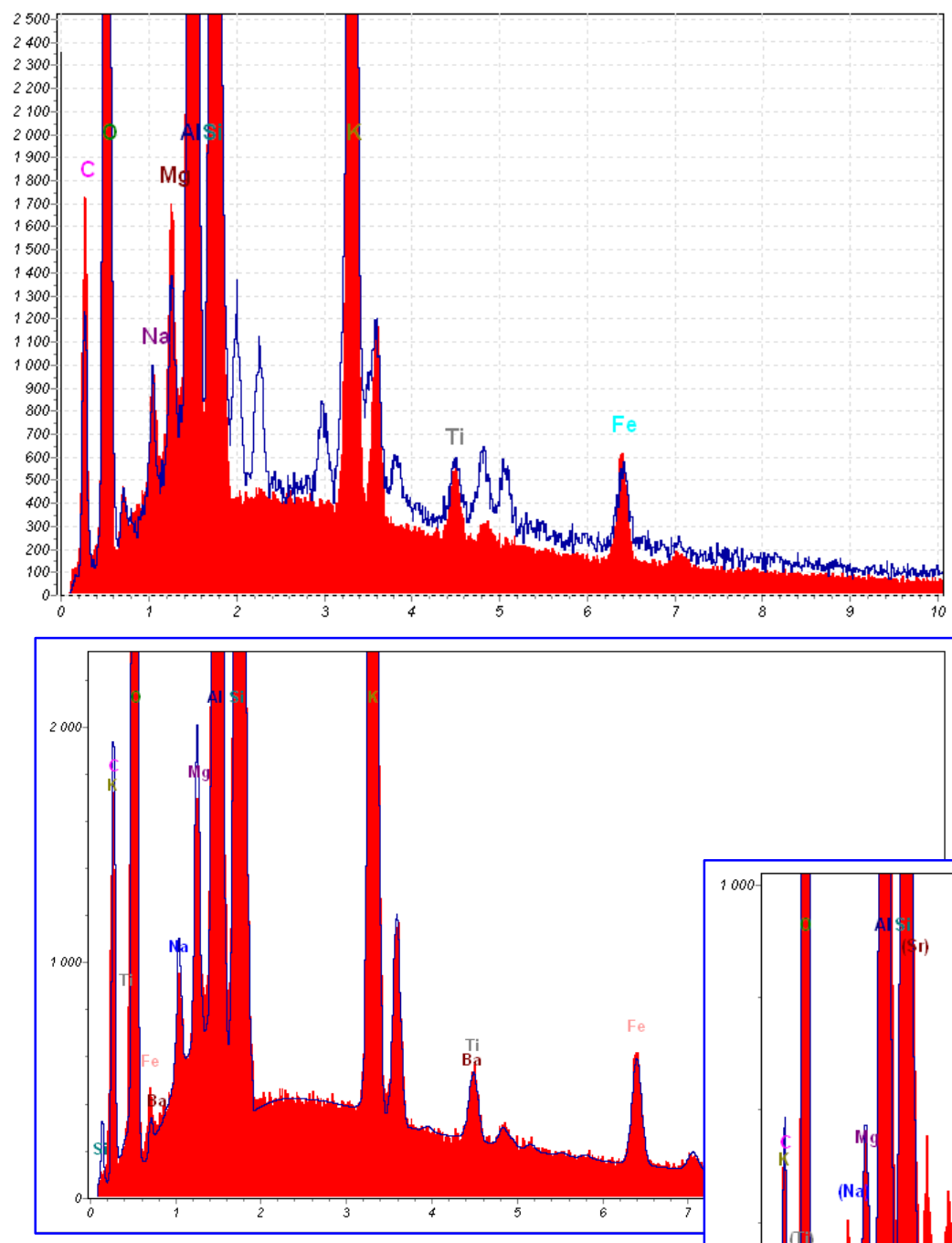

Almost same results of EXpertID with evaluation of the $209 \mathrm{kcps}$ Mica spectrum, because Smart PileUp Consideration is always using a hidden pile-up corrected spectrum for evaluation.

FIG. 1.

Mica spectrum with $4 \mathrm{kcps}$ (red bar spectrum) vs. $209 \mathrm{kcps}$ (blue line spectrum) with visible differences in entire X-ray distribution (normalized to total spectrum counts).

FIG. 2.

EXpertID results with $4 \mathrm{kcps}$ Mica $\leftarrow$ spectrum

(reconstructed spectrum: blue line) 